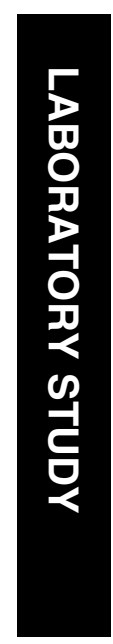

\section{Do scleral flap dimensions influence reliability of intraocular pressure control in experimental trabeculectomy?}

${ }^{1}$ Wellington Hospital, Wellington, New Zealand

${ }^{2}$ Wellington School of Medicine, Wellington, New Zealand

Correspondence: W Birchall, Eye Department, Wellington Hospital, Riddiford Street, Wellington, New Zealand Tel: + 634385 5999; Fax: + 6343855470 . E-mail: w.birchall@ xtra.co.nz

Received: 11 April 2005 Accepted in revised form: 6 December 2005 Published online: 27 January 2006

Financial Support: This work was supported by the Capital Vision Research Trust

Proprietary interests: None

\begin{abstract}
Aim To compare the effect on intraocular pressure (IOP) of large $v$ s small scleral flap size during trabeculectomy using adjustable sutures

Methods Trabeculectomy operations were performed on nine donor human eyes connected to a constant flow infusion with real-time IOP monitoring. Large scleral flaps $\left(4 \times 4 \mathrm{~mm}, 16 \mathrm{~mm}^{2}, n=12\right)$ or small scleral flaps $\left(3 \times 2 \mathrm{~mm}, 6 \mathrm{~mm}^{2}, n=9\right)$ were constructed over $0.76 \mathrm{~mm}^{2}$ sclerostomies. For each procedure, equilibrium IOP was measured following tight closure with two four-throw adjustable 10-0 nylon sutures.
\end{abstract}

Results Five scleral flaps were thin or poorly constructed; four of these were in the initial seven procedures, implying learning effect. These had a mean absolute IOP of $7.6 \mathrm{mmHg}$

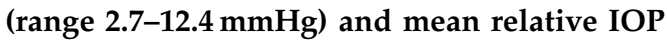
of $28.3 \%$ of baseline (range $10-45.8 \%$ ) after closure. In the remaining 16 good quality procedures, mean IOP was $1.3 \mathrm{mmHg}$ (range 0-3.4 mmHg) after sclerostomy, confirming minimal outflow resistance before closure. Following flap closure mean IOP was $20 \mathrm{mmHg}$ (SD 4.4, range 15.5-29.3 $\mathrm{mmHg}$ ) for large $(n=8)$, and $18.7 \mathrm{mmHg}$ (SD 3.6, 15.9-25.8 $\mathrm{mmHg})$ for small $(n=8)$ flaps (unpaired $\boldsymbol{t}$-test, $\boldsymbol{P}=\mathbf{0 . 2 6}$ ). Mean IOP (\% baseline) was $71.6 \%$ (SD 8.4, range $60.6-86.6 \%$ ) and $66 \%(\mathrm{SD}=12.7,46.8-86.6 \%)$ for large and small flap groups, respectively (unpaired $t$ test, $P=0.2$ ).

Conclusions Well-constructed scleral flaps of both sizes were able to support an average IOP at least two-thirds of baseline, and both had similar absolute IOP levels. Errors in flap construction resulted in loss of IOP control. Smaller flap size does not appear to compromise control of early postoperative IOP using adjustable sutures.

Eye (2007) 21, 402-407 doi:10.1038/sj.eye. 6702253; published online 27 January 2006

Keywords: glaucoma; trabeculectomy; surgical technique; adjustable sutures; experimental; intraocular pressure

\section{Introduction}

Trabeculectomy, or guarded filtration surgery, was developed $^{1,2}$ because the presence of a scleral flap might avoid the complications of full-thickness surgery. The creation of a partial thickness scleral flap overlying the internal sclerostomy improves control of aqueous outflow compared with 'full-thickness' procedures such as the Scheie technique. With control over aqueous flow, the risks of hypotony and its sequelae: anterior chamber shallowing, blood aqueous barrier breakdown (with potential for increased healing response and failure by scarring), choroidal effusions, hypotony maculopathy, cataract, hyphaema, suprachoroidal haemorrhage, and aqueous misdirection ${ }^{3,4}$ can be minimised. The relatively high frequency of these complications relating to early postoperative IOP control ${ }^{5,6}$ that has been a significant factor in the development of alternative nonpenetrating surgical techniques. These have taught us that control of the IOP in the first days after surgery reduces risk of hypotony and its complications, although evidence from comparative studies to date ${ }^{7,8}$ suggest that IOP outcomes are superior following trabeculectomy. 
Following the description of the original trabeculectomy technique, ${ }^{1}$ which combined a large $5 \times 5 \mathrm{~mm}$ rectangular partial thickness scleral flap with excision of a $1 \times 4 \mathrm{~mm}$ long block of trabecular tissue, various modifications have been applied in an attempt to reduce complications and maintain adequate long-term intraocular pressure (IOP) control. ${ }^{9-15}$ These include variation in the shape ${ }^{11}$ and size $\mathrm{e}^{12,15}$ of the scleral flap and internal sclerostomy, ${ }^{10,12}$ and tightness of scleral flap closure. ${ }^{13,14}$ None of these modifications have been demonstrated to improve long term IOP control, which is largely determined by the healing response of the subconjunctival tissues, ${ }^{16}$ but trabeculectomy complication rates ought to be decreased significantly by techniques that can reliably assert control over aqueous flow in the early postoperative phase.

Relatively tight scleral flap closure may reduce the risk of early hypotony ${ }^{13}$ and there is evidence that manipulation of adjustable scleral flap sutures allows more reliable postoperative adjustment of IOP than suture removal, suture lysis or ocular massage. ${ }^{17}$ This is especially beneficial in Mitomycin C enhanced trabeculectomy where low IOP in the first two postoperative weeks is a risk factor for more prolonged hypotony. ${ }^{18}$

Trabeculectomy using a small scleral flap appears to provide medium to long-term IOP control comparable to large flap techniques ${ }^{12,15,19}$ and may offer potential advantages: reduced surgical tissue trauma, a larger area of undisturbed sclera and conjunctiva should repeat surgery be required, and reduced astigmatism induction. ${ }^{20}$ Potential disadvantages include possible decreased control over aqueous flow, technical challenges including difficulty in dissecting a large treatment area for antimetabolite application and difficulty in controlling subconjunctival bleeding if the conjunctival incision is small. Larger scleral flaps might give better tissue apposition over the scleral bed, and may give better IOP control. It may also be more difficult to create either large or small scleral flaps with a high degree of reproducibility for technical reasons.

As far as we are aware, there has been no research into the effects of scleral flap dimensions on aqueous dynamics and intraoperative IOP control in trabeculectomy. We compared the effects of large vs small scleral flaps secured with adjustable sutures while making real-time measurements of IOP in an experimental steady-state infusion model. ${ }^{17,21,22}$

\section{Methods}

Nine grossly normal phakic human donor eyes with no history of previous intraocular surgery were available for study. Normal saline was injected into the posterior chamber using a $29 \mathrm{G}$ needle through the optic nerve to reinflate the globe. The anterior chamber was intubated with a 25G intravenous cannula, using a long corneal path to avoid paracannular leakage. The cannula was attached with nonexpansile tubing to a continuous flow syringe pump (Harvard Apparatus, www.harvardapparatus.com) and a Powerlab real-time IOP monitoring system (AD Instruments Pty Ltd, Castle Hill NSW 2154, Australia, www.adinstruments.com) (Figure 1).

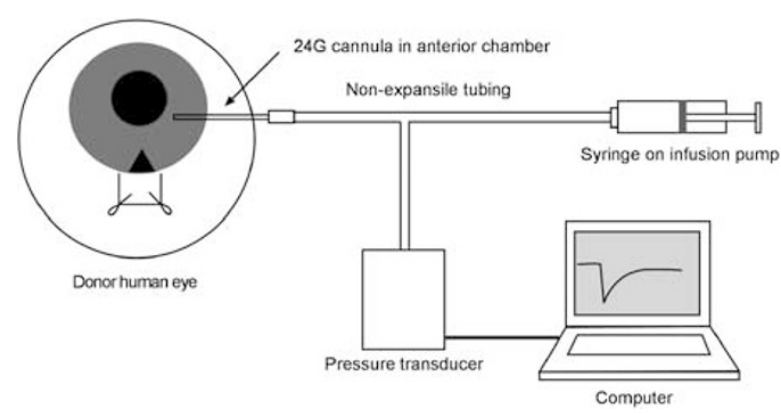

Figure 1 Experimental setup. The syringe pump (right) provides a constant flow of balanced salt solution (BSS) into the eye under test. The intraocular pressure is measured via a transducer attached to a personal computer (PC) for data acquisition and analysis.

The IOP sensor was calibrated and zeroed with respect to atmospheric pressure prior to initiation of each trabeculectomy site. An infusion via the system was then commenced and flow rate required to keep the IOP of the eye under investigation between 23 and $32 \mathrm{mmHg}$ determined. The infusion rate varied from eye to eye and ranged from 10 to $18 \mathrm{microl} / \mathrm{min}$.

After the IOP was stable, conjunctiva and loose episcleral tissue was removed from one quadrant to prepare a site for the procedure. Initially, a half thickness incision was made behind and tangential to the limbus using a $0.3 \mathrm{~mm}$ guarded blade (Beaver) and this incision converted to a scleral tunnel with a crescent blade (Alcon, Fort Worth, TX, USA). The sides of the scleral flap were cut with a $45^{\circ}$ blade (Alcon, Fort Worth, TX, USA) up to the limbus. The anterior chamber was entered using a $15^{\circ}$ blade (Alcon, Fort Worth, TX, USA) to make a $2 \mathrm{~mm}$ centrally placed slit in the peripheral cornea under the base of the scleral flap. A $1 \mathrm{~mm}$ trabeculectomy punch (Moria, www.moria-surgical.com) was used to remove a central hemispherical block of corneotrabecular tissue (area $0.76 \mathrm{~mm}^{2}$ ) and a peripheral iridectomy performed with scissors. Two adjustable sutures were placed at the corners of the flap after passing through the cornea to mimic their normal position and force vectors. Four throws were placed on each suture, which were gradually tightened alternately 


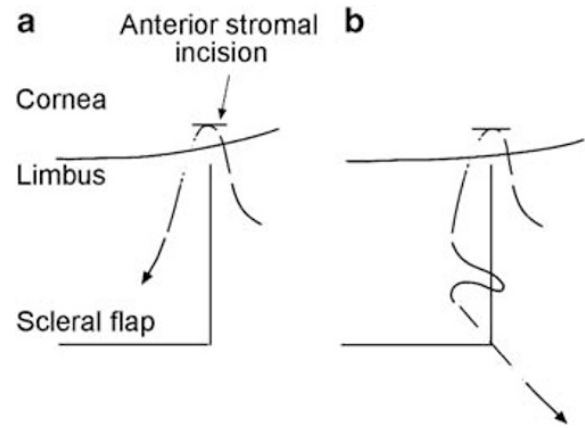

C

Suture tying forceps

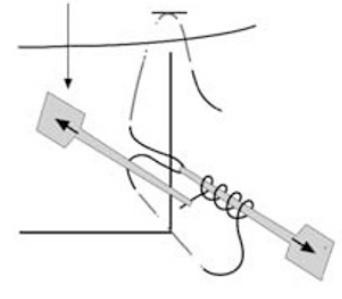

d

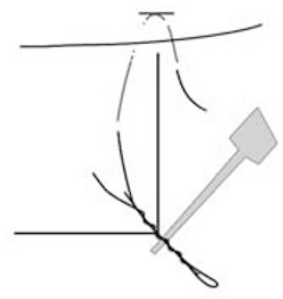

Figure 2 Adjustable suture insertion technique (right side of scleral flap illustrated). The suture is passed superficially across the limbus exiting through a preplaced paralimbal superficial anterior stromal incision and then passed back superficially through the scleral flap (a). A third pass is placed through the corner of the flap and corner of the scleral bed leaving a small loop of suture over the scleral flap (b). The suture is tied with four throws pulled over this loop (c, d). Suture tension may be reduced (clinically, through the conjunctiva) by manipulating the knot with forceps (d). The suture may also be removed by retrieval and pulling of the peripheral corneal subepithelial suture loop.

to ensure the scleral flap was well positioned and sitting symmetrically within its bed (Figures 2). Elsewhere, residual conjunctiva was left in place and the scleral surface coated with viscoelastic to prevent scleral dehydration.

Two sizes of scleral flap were created in otherwise identical procedures - either a large $4 \times 4 \mathrm{~mm}$ scleral flap (area $16 \mathrm{~mm}^{2}$ ) or a small $3 \times 2 \mathrm{~mm}$ scleral flap (area $6 \mathrm{~mm}^{2}$ ) (Figure 3). For the smaller scleral flap, the dimensions of $3 \times 2 \mathrm{~mm}$ were chosen as this allowed formation of a scleral tunnel with a commercially available crescent blade, facilitating creation of a flap of even thickness and mimicking the larger flap procedure. The longer $(3 \mathrm{~mm})$ side was placed tangential to the limbus. Flap dimensions were accurately measured with preset callipers the tips of which were used to indent the sclera to mark the ends of the initial tangential scleral incision.

The IOP was then allowed to reach equilibrium while maintaining the initial infusion rate, and the equilibrium IOP recorded. The time to reach equilibrium varied from 4.2 to $8.4 \mathrm{~min}$ (mean $6.3 \mathrm{~min}$ ) for all flaps. The two sutures were then untied and the equilibrium IOP for the unsutured flap recorded.

If there was sufficient space for another trabeculectomy and the condition of the eye under investigation was suitable without visible scleral dehydration, the scleral flap was closed tightly by retying the original sutures and placing additional sutures to close the three edges of the flap, the surface of the scleral flap dried and cyanoacrylate adhesive applied to the flap edges to seal the wound. If the IOP could be maintained above $23 \mathrm{mmHg}$ at the initial infusion rate the above steps were repeated at a new site. The number of study trabeculectomies on each eye varied between one and three (Table 1). All procedures were performed by a

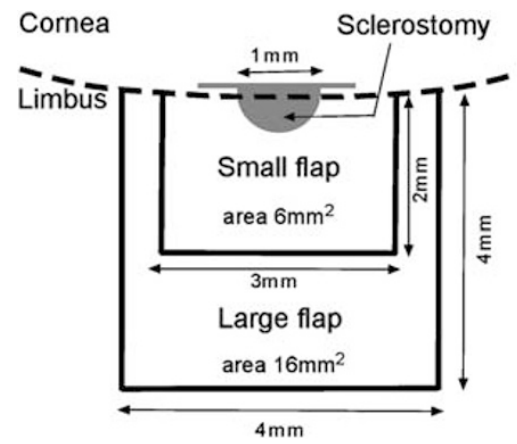

Figure 3 Diagram showing the relative proportions of the two flap sizes used and position relative to the internal sclerostomy.

glaucoma fellow experienced in the clinical application of the surgical technique used in the study.

To ensure consistency in scleral flap formation and satisfactory positioning after suturing, the following subjective criteria were adopted to confirm technical 'adequacy': (1) a scleral flap of between one-half and two-thirds thickness in its entirety; (2) a centrally placed sclerostomy with a diameter no greater than $1 \mathrm{~mm}$; and (3) following suture tying, a symmetrically placed scleral flap with each corner well seated into the corresponding corner of the scleral bed.

Data were collated in Microsoft Excel Spreadsheets and statistical analysis performed using Excel data analysis software. The unmatched, two-tailed $t$-test was used to assess differences between the two groups.

\section{Results}

Of the 21 procedures performed on nine eyes (Table 1), four large flap procedures and a single small flap procedure were judged technically 'poor' for one of the following reasons: (1) very thin flap, (2) small 
Table 1 Summary of outcomes for each trabeculectomy procedure

\begin{tabular}{|c|c|c|c|c|c|c|}
\hline Eye no. & Trab no. & Flap dimensions & Technically adequate? & Baseline IOP & Post sclerostomy IOP & Post suture IOP (\% of baseline) \\
\hline \multirow[t]{4}{*}{1} & 1 & $4 \times 4$ & Yes & 30 & 1.1 & 66.7 \\
\hline & 2 & $3 \times 2$ & $\mathrm{No}^{\mathrm{a}}$ & 30.3 & 0.8 & 40.9 \\
\hline & 3 & $4 \times 4$ & $\mathrm{No}^{\mathrm{b}}$ & 27 & 0.7 & 10 \\
\hline & 4 & $3 \times 2$ & Yes & 27.8 & 1.9 & 57.2 \\
\hline \multirow[t]{2}{*}{2} & 5 & $4 \times 4$ & $\mathrm{No}^{c}$ & 26.8 & 0.2 & 18.7 \\
\hline & 6 & $4 \times 4$ & Yes & 27 & 1.2 & 78.5 \\
\hline \multirow[t]{2}{*}{3} & 8 & $4 \times 4$ & $\mathrm{No}^{\mathrm{a}}$ & 24 & 1.3 & 11 \\
\hline & 7 & $3 \times 2$ & Yes & 28 & 0.9 & 71.44 \\
\hline \multirow[t]{2}{*}{4} & 9 & $3 \times 2$ & Yes & 32 & 0 & 46.8 \\
\hline & 10 & $3 \times 2$ & Yes & 28 & 0.6 & 77.5 \\
\hline \multirow[t]{3}{*}{5} & 11 & $4 \times 4$ & Yes & 25 & 0 & 68 \\
\hline & 12 & $3 \times 2$ & Yes & 26.3 & 1.4 & 63 \\
\hline & 13 & $4 \times 4$ & Yes & 28 & 0 & 70.7 \\
\hline \multirow[t]{2}{*}{6} & 14 & $3 \times 2$ & Yes & 24.2 & 3.4 & 64.8 \\
\hline & 15 & $4 \times 4$ & Yes & 24 & 1.7 & 65 \\
\hline \multirow[t]{3}{*}{7} & 16 & $4 \times 4$ & Yes & 28 & 0.5 & 77.1 \\
\hline & 17 & $3 \times 2$ & Yes & 29.8 & 2.7 & 86.6 \\
\hline & 18 & $4 \times 4$ & Yes & 32 & 2.7 & 86.2 \\
\hline \multirow[t]{3}{*}{8} & 19 & $4 \times 4$ & $\mathrm{No}^{\mathrm{d}}$ & 27 & -0.2 & 25.9 \\
\hline & 20 & $3 \times 2$ & Yes & 31.7 & 0.3 & 56.8 \\
\hline & 21 & $4 \times 4$ & Yes & 25.8 & 1 & 60.6 \\
\hline
\end{tabular}

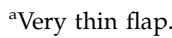

bLarge irregular sclerostomy.

cPoorly seated flap at one corner.

${ }^{\mathrm{d}}$ Very thin ragged flap near limbus on one side.

sclerostomy enlarged resulting in an irregular

asymmetric fistula close to one edge of the scleral flap, (3) one scleral flap corner not sitting well into scleral bed despite suture replacement, with some cheese-wiring of the suture, or (4) scleral flap very thin near limbus on one side with irregular flap edge. These poor quality flaps resulted in a mean absolute IOP of $7.6 \mathrm{mmHg}$ (range $2.7-12.4 \%$ ) and a mean relative IOP of $28.3 \%$ of baseline (range 10-45.8\%) following suture closure.

In the remaining 16 good quality procedures in the nine eyes, mean IOP was $1.3 \mathrm{mmHg}$ (range $0-3.4 \mathrm{mmHg}$ ) after sclerostomy, confirming minimal outflow resistance before closure. Following flap closure mean IOP was $20 \mathrm{mmHg}$ (SD 4.4, range $15.5-29.3 \mathrm{mmHg}$ ) for large $(n=8)$, and $18.7 \mathrm{mmHg}$ (SD 3.6, range $15.9-25.8 \mathrm{mmHg}$ ) for small $(n=8)$ flaps $(P=0.26)$. Mean IOP (\% of baseline) was 71.6\% (SD 8.4, range 60.6-86.6\%) and 66\% (SD 12.7, range $46.8-86.6 \%)$ for large and small flap groups, respectively $(P=0.2)$.

Baseline IOP, IOP following sclerostomy, and equilibrium IOP following suture closure of the scleral flap for each procedure are displayed in Table 1 and summarised in Figure 4.

For all procedures, regardless of technical 'adequacy', mean IOP was $55 \%$ of baseline for the large scleral flap group and $64 \%$ for the small scleral flap group. For technically good procedures, mean IOP was $71.6 \%$ of baseline for the large scleral flap group, and $66 \%$ of

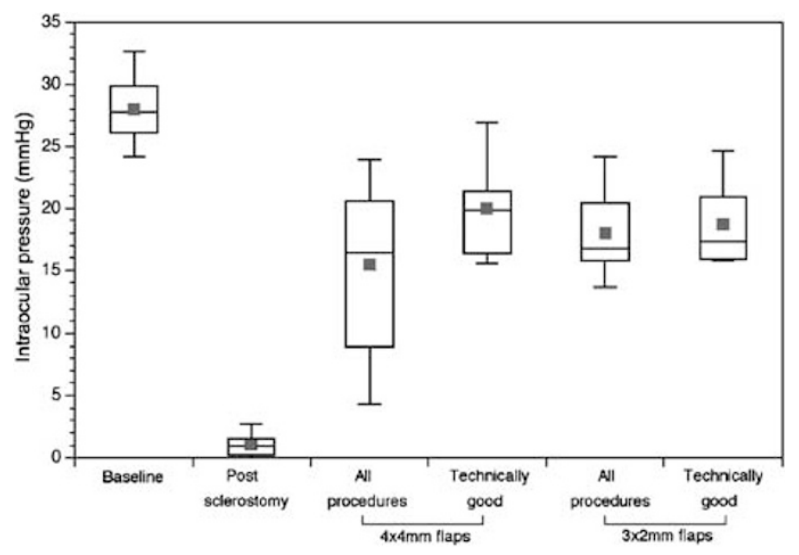

Figure 4 Boxplot showing range of IOP at baseline, after sclerostomy and after suture tying for both scleral flap sizes.

baseline for the small scleral flap group $(P=0.2)$

(Figure 5).

\section{Discussion}

Following trabeculectomy surgery, egress of aqueous from the eye beneath a well-constructed scleral flap will depend on a number of factors. The principle resistance to aqueous outflow from the eye via the sclerostomy will be determined by the apposition of the flap to the underlying scleral bed around the sclerostomy and its 


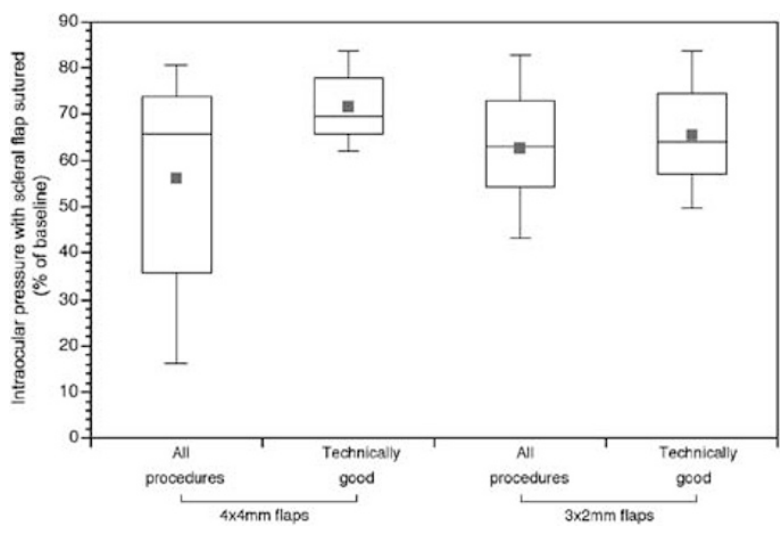

Figure 5 Boxplot showing intraocular pressure (\% of baseline) for each scleral flap size.

ability to lift. This will depend upon flap elasticity, which will be influenced by flap thickness, and tension within the flap determined by suture position and tension. This study cannot address wound healing or long-term outcomes, but only the potential for tight control over the early postoperative IOP by scleral flap construction in conjunction with previous work describing manipulation of suture tension.

The small $3 \times 2 \mathrm{~mm}$ scleral flap used here was slightly larger than those in published series of small flap or 'micro' trabeculectomy. In the initial report of small flap trabeculectomy, Starita et al ${ }^{12}$ compared a small $2 \times 2 \mathrm{~mm}$ flap procedure in 17 eyes with a large $4 \times 4 \mathrm{~mm}$ flap procedure in 11 eyes, with similar IOP control at 1 year in the 2 groups. In this study, the larger flap procedures had a larger sclerostomy (4vs $\left.1 \mathrm{~mm}^{2}\right)$.

In a retrospective study of 'microtrabeculectomy' Vernon and Spencer used small $2 \times 2 \mathrm{~mm}$ square flaps on 65 eyes-mean IOP at 13 months was $13.4 \mathrm{mmHg}$ with $88 \%$ of eyes controlled at $<21 \mathrm{mmHg}$ on no medications ${ }^{15}$ - results comparable to published studies of conventional large flap techniques with similar follow up. ${ }^{23,24}$ In our study, the ratio of scleral flap area to sclerostomy area was similar (15 vs 18) as we used a slightly larger diameter sclerostomy punch (1 vs $0.75 \mathrm{~mm}$ ). This ratio was significantly larger than in the study of Starita et al ${ }^{12}$ (15 vs 4). However, the size of sclerostomy does not appear to increase the likelihood of postoperative flat anterior chamber or choroidal detachment, nor influence long term control of IOP. ${ }^{10}$

This study had relatively small numbers, reflecting the difficulty in procuring human donor eyes. Although the only variable was scleral flap size there were likely to be small differences in scleral flap thickness, suture positioning, and suture tension within each group, influencing the final IOP. The advantage of using adjustable sutures of this type for this study is that they were likely to give better control and reproducibility of suture tension than, for example, conventional 3/1/1 throw fixed sutures, where the second throw may shorten the knot and suture loop considerably with resulting increase in suture and scleral flap tension. Adjustable four-throw sutures probably have an upper limit of generated tension.

All of our procedures with good IOP control had flaps judged to be at least half scleral thickness, and both procedures with very thin flaps (one in each size group) resulted in increased filtration and poorer IOP maintenance. While some outflow through the flap may have occurred, increased gaping of the edges of one very thin $4 \times 4 \mathrm{~mm}$ flap was noted, despite well-positioned and -seated flap corners, suggesting that reduced flap rigidity and loss of resistance to lifting were the main factors causing increased outflow. The clinical implication is that should a thin scleral flap be created, then adjustable sutures of this type may not create sufficient flap tension to control outflow and it might be advisable to improve flap closure with additional sutures to reduce the risk of hypotony. Very thin or perforated scleral flaps may not be capable of maintaining a safe IOP at all, regardless of suture application.

The five procedures classed as technically poor all produced an IOP significantly lower than procedures that were felt to be satisfactory, and illustrate the importance of meticulous surgical technique in glaucoma filtration surgery to ensure consistent postoperative outcomes. The issues of surgical exposure and learning effects have been discussed recently in the context of the declining numbers of glaucoma operations. ${ }^{25}$ The UK National Trabeculectomy Study ${ }^{26}$ highlights the importance of adequate supervision and training. Nonconsultant career grade surgeons achieved lower success rate than other surgeons groups, but no difference was found between junior ophthalmology trainees and fully qualified surgeons. This may reflect differences in case selection and increased supervision and teaching provided to trainees.

Although the numbers were small, this study suggests that well-constructed scleral flaps measuring either $3 \times 2$ or $4 \times 4 \mathrm{~mm}$ were able to support an average IOP at least two-thirds of baseline, and both produced similar absolute IOP levels. Errors in flap construction resulted in loss of IOP control; these were more common in the large flap group, and largely occurred early in the series. Smaller flap size does not appear to compromise control of early postoperative IOP provided by adjustable sutures, suggesting the techniques may be usefully combined in clinical practice. Further studies into the hydrodynamics of scleral flap function and optimal trabeculectomy construction may lead to increased predictability and safety of our most common and successful glaucoma operation. 


\section{References}

1 Cairns JE. Trabeculectomy. Preliminary report of a new method. Am J Ophthalmol 1968; 66: 673-679.

2 Watson PG, Grierson I. The place of trabeculectomy in the treatment of glaucoma. Ophthalmology 1981; 88: 175-196.

3 Spaeth GL, Joseph NH, Fernandes E. Trabeculectomy: a reevaluation after three years and a comparison with Scheie's procedure. Ophthalmic Surg 1975; 6: 27-38.

4 Borisuth NS, Phillips B, Krupin T. The risk profile of glaucoma filtration surgery. Curr Opin Ophthalmol 1999; 10 112-116.

5 Khaw PT, Wells AP, Lim KS. Surgery for glaucoma. Br J Ophthalmol 2003; 87: 517.

6 Edmunds B, Thompson JR, Salmon JF, Wormald RP. The national survey of trabeculectomy. III. Early and late complications. Eye 2002; 16: 297-303.

7 Netland PA. Nonpenetrating glaucoma surgery. Ophthalmology 2001; 108: 416-421.

8 O'Brart DP, Shiew M, Edmunds B. A randomised, prospective study comparing trabeculectomy with viscocanalostomy with adjunctive antimetabolite usage for the management of open angle glaucoma uncontrolled by medical therapy. Br J Ophthalmol 2004; 88: 1012-1017.

9 Stewart RH, Kimbrough RL, Bachh H, Allbright M. Trabeculectomy and modifications of trabeculectomy. Ophthalmic Surg 1979; 10: 76-80.

10 Duzanec Z, Krieglstein GK. The relationship between pressure regulation and anatomic localization as well as trephine sites in goniotrepanation. A prospective study. Klin Monatsbl Augenheilkd 1981; 178: 431-435.

11 Kimbrough RL, Stewart RH, Decker WL, Praeger TC. Trabeculectomy: square or triangular scleral flap? Ophthalmic Surg 1982; 13: 753.

12 Starita RJ, Fellman RL, Spaeth GL, Poryzees EM. Effect of varying size of scleral flap and corneal block on trabeculectomy. Ophthalmic Surg 1984; 15: 484-487.

13 Batterbury M, Wishart PK. Is high initial aqueous outflow of benefit in trabeculectomy? Eye 1993; 7: 109-112.

14 Bluestein EC, Stewart WC. Tight vs loose scleral flap closure in trabeculectomy surgery. Doc Ophthalmol 1993; 84: 379-385.
15 Vernon SA, Spencer AF. Intraocular pressure control following microtrabeculectomy. Eye 1995; 9 : 299-303.

16 Khaw PT, Chang L, Wong TT, Mead A, Daniels JT, Cordeiro MF. Modulation of wound healing after glaucoma surgery. Curr Opin Ophthalmol 2001; 12 143-148.

17 Wells AP, Bunce C, Khaw PT. Flap and suture manipulation after trabeculectomy with adjustable sutures: titration of flow and intraocular pressure in guarded filtration surgery. J Glaucoma 2004; 13: 400-406.

18 Hara T, Araie M, Shirato S, Yamamoto S. Conditions for balance between lower normal pressure control and hypotony in mitomycin trabeculectomy. Graefes Arch Clin Exp Ophthalmol 1998; 236: 420-425.

19 Vernon SA, Gorman C, Zambarakji HJ. Medium to longterm intraocular pressure control following small flap trabeculectomy (microtrabeculectomy) in relatively low risk eyes. Br J Ophthalmol 1998; 82: 1383-1386.

20 Vernon SA, Zambarakji HJ, Potgieter F, Evans J, Chell PB. Topographic and keratometric astigmatism up to 1 year following small flap trabeculectomy (microtrabeculectomy). Br J Ophthalmol 1999; 83: 779-782.

21 Eisenberg DL, Koo EY, Hafner G, Schuman JS. In vitro flow properties of glaucoma implant devices. Ophthalmic Surg Lasers 1999; 30: 662-667.

22 Lim KS, Wells AP, Khaw PT. Needle perforations of Molteno tubes. J Glaucoma 2002; 11: 434-438.

23 Mills KB. Trabeculectomy: a retrospective long-term follow-up of 444 cases. Br J Ophthalmol 1981; 65: 790-795.

24 Jay JL, Murray SB. Early trabeculectomy vs conventional management in primary open angle glaucoma. $\mathrm{Br} \mathrm{J}$ Ophthalmol 1988; 72: 881-889.

25 Whittaker KW, Gillow JT, Cunliffe IA. Is the role of trabeculectomy in glaucoma management changing? Eye 2001; 15: 449-452.

26 Edmunds B, Bunce CV, Thompson JR, Salmon JF, Wormald RP. Factors associated with success in first-time trabeculectomy for patients at low risk of failure with chronic open-angle glaucoma. Ophthalmology 2004; 111: 97-103. 